\title{
Design for AC servo position loop based on RBF neural network predictive control
}

\author{
Ying Zhang \\ Department of physics and information engineering \\ Jining University \\ Qufu, China \\ zhangyingcad@126.com
}

Ke Zhou

Department of physics and information engineering Jining University

Qufu, China

zhouke81@163.com

\author{
Yongguang Gong \\ Department of physics and information engineering \\ Jining University \\ Qufu, China \\ mm77nn88@163.com
}

\section{Lifeng Chen}

Department of physics and information engineering Jining University

Qufu, China

columbus1980@126.com

\begin{abstract}
Aiming at wide variations in loads and moment of inertia of the machine tool position servo system, the position loop controller is designed based on RBF neural network control and predictive control. The mathematical model of AC PMSM is established. The predictive model is designed based on controlled autoregressive integral moving average model, obtained the predictive vector and the reference trajectory. The RBF neural network structure is established, tuning PID algorithm is designed. A new control strategy which combined with predictive control and RBF neural network PID control is obtained. Compared with the torque fluctuation, anti-interference ability and tracking properties of the traditional PID control, predictive control ensures that the system tracking performance and RBF neural network control can adjust PID parameters on-line, which guarantees the robustness in the external disturbance and parameter perturbation. The simulation results demonstrate that the RBF neural network predictive controller can guarantee the static and dynamic performance of the system.
\end{abstract}

Keywords- RBF neural network; predictive control; AC PMSM; machine tool; position control

\section{Introduction}

The machine tool servo drive actuator is mainly permanent magnet synchronous motor. It mainly consists of the current loop, the speed loop and the position loop. In the traditional machine tool servo control system, PID controller is often used in the speed loop and position loop control. Due to the traditional PID control without considering time-varying, nonlinear and uncertainty of the model parameters, the control effect need to improve ${ }^{[1-2]}$.

In recent years, the RBF neural network and predictive control have more applications in AC servo system. The advantages of predictive control are the diversity of the predictive model, rolling optimization scheduling and online calibration adaptability ${ }^{[3-4]}$. RBF neural network control is simple, and its weighting coefficient can be real-time adjustment ${ }^{[5-7]}$.
RBF neural network predictive controller is designed. Predictive control ensures that the system tracking performance. RBF neural network can adjust PID parameters on-line and restrain the system parameter change and load disturbance. Simulation results show that the designed controller has better robustness.

\section{THE MATHEMATICAL MODEL OF MOTOR}

Assumes: the permanent magnet synchronous motor is not saturated, the eddy current and hysteresis losses can be ignored, under the synchronous rotating coordinate system, the stator $\mathrm{d}, \mathrm{q}$ axis voltage equation is as follows [8-10]:

$$
\left\{\begin{array}{l}
U_{d}=R i_{d}+p_{n} L_{d} i_{d}+p_{n} \varphi_{f}-\omega \Psi_{q} \\
U_{q}=R i_{q}+p_{n} L_{q} i_{q}+\omega \Psi_{d}
\end{array}\right.
$$

Where $L_{d}$ and $L_{q}$ are the synchronous inductance of $d q$ axis $\left(L_{d}=L_{q}=L\right), \omega$ is the motor speed, $\Psi_{d}$ and $\Psi_{q}$ are the magnetic chain of the dq axis, $\varphi_{f}$ is the rotor flux linkage, $\mathrm{R}$ is the stator winding resistance.

According to vector control, the motor torque is as follows:

$$
T_{e}=\frac{3}{2} p_{n} \varphi_{f} i_{q}=K_{t} i_{q}
$$

$K_{t}$ is constant torque, $p_{n}$ is motor poles logarithmic.

In general, permanent magnet servo motor mechanical equation expressed as follows:

$$
T_{e}=J \dot{\omega}+T_{L}+B \omega
$$

$J$ is the moment of inertia, $B$ is the coefficient of friction, $T_{L}$ is the load torque.

Substituting (2) into (3), the mechanical dynamic equation of permanent magnet synchronous motor is as follows: 


$$
\ddot{\theta}_{\mathrm{r}}=-\frac{B}{J} \dot{\theta}_{r}+\frac{K_{t}}{J} i_{q}-\frac{T_{L}}{J}
$$

$\theta_{r}$ is rotor angle, $\theta_{r}=\omega$.

\section{CONTROLler Design}

\section{A. Design for predictive controller}

The predictive model is using controlled autoregressive integral moving average model. Model is as follows:

$$
\mathrm{y}(k)=\frac{z^{-1} B}{A} u(k)+\frac{C}{A \Delta} \xi(k)
$$

$\mathrm{A}$ and $\mathrm{C}$ are $\mathrm{n}$ order polynomial of $\mathrm{z}^{-1}, \mathrm{~B}$ is $\mathrm{m}$ order polynomial of $\mathrm{z}^{-1}, \mathrm{n}$ is the maximum prediction length, $\mathrm{m}$ is control length.

Predictive vector $f$ :

$$
\mathrm{f}=H \Delta u(k)+F y(k)
$$

$H$ and $F$ are prediction adjustment matrix.

The reference trajectory $W$ follows:

$$
W=Q y(k)+M e(k)
$$

$e(k)$ is error signal,

$$
\begin{aligned}
& W=[w(k+1), w(k+2), \cdots, w(k+n)]^{T} \\
& Q=\left[\alpha, \alpha^{2}, \cdots, \alpha^{n}\right]^{T} \\
& M=\left[1-\alpha, 1-\alpha^{2}, \cdots, 1-\alpha^{n}\right]^{T}
\end{aligned}
$$

Predictive control closed-loop structure is shown in Fig.1.

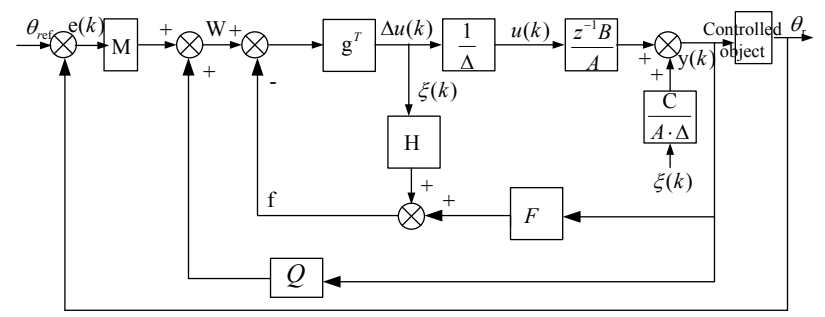

Figure 1. Predictive control closed loop structure

The closed-loop transfer function is as follows:

$$
\frac{y(k)}{y_{r}}=\frac{z^{-1} B g^{T} M}{\left(1+g^{T} H\right) A \Delta+z^{-1} B g^{T}(F-Q)}
$$

The output response of the closed loop system is as follows:

$$
\begin{aligned}
y(k)= & \frac{z^{-1} B g^{T} M}{\left(1+g^{T} H\right) A \Delta+z^{-1} B g^{T}(F-Q)} y_{r}+ \\
& \frac{\left(1+g^{T} H\right) C}{\left(1+g^{T} H\right) A \Delta+z^{-1} B g^{T}(F-Q)} \xi(k)
\end{aligned}
$$

\section{B. RBF neural network PID position controller}

1) $R B F$ neural network structure

$\mathrm{RBF}$ network is three layers forward networks. The network structure is shown in Fig.2.

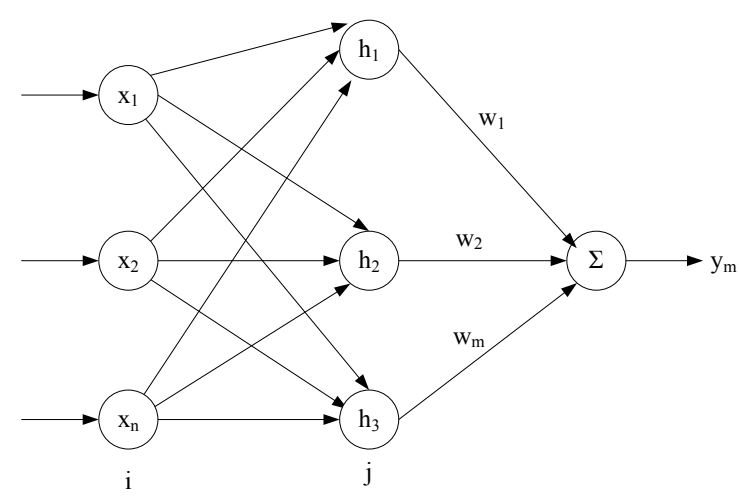

Figure 2. RBF neural network structure

2) The Jacobian information identification algorithm of controlled object

According to the gradient descent method, output weights, node center, and node basis width parameters are as follows:

$$
\left\{\begin{array}{l}
\Delta \mathrm{w}_{\mathrm{j}}(k)=\eta\left(y(k)-y_{m}(k)\right) h_{j} \\
w_{j}(k)=w_{j}(k-1)+\Delta \mathrm{w}_{\mathrm{j}}(k)+\alpha\left(w_{j}(k-1)-w_{j}(k-2)\right) \\
\Delta b_{j}(k)=\eta\left(y(k)-y_{m}(k)\right) w_{j} h_{j} \frac{\left\|X-C_{j}\right\|^{2}}{b_{j}^{3}} \\
b_{j}(k)=b_{j}(k-1)+\Delta b_{j}+a\left(b_{j}(k-1)-b_{j}(k-2)\right) \\
\Delta c_{j i}(k)=\eta\left(y(k)-y_{m}(k)\right) w_{j} \frac{x_{j}-c_{j i}}{b_{j}{ }^{2}} \\
c_{j i}(k)=c_{j i}(k-1)+\Delta c_{j i}(k)+\alpha\left(c_{j i}(k-1)-c_{j i}(k-2)\right)
\end{array}\right.
$$

Where $\eta$ is learning rate, $\alpha$ is Momentum factor. Jacobian matrix algorithm is as follows:

$$
\frac{\partial \mathrm{y}(k)}{\partial \Delta u(k)} \approx \frac{\partial y_{m}(k)}{\partial \Delta u(k)}=\sum_{j=1}^{m} w_{j} h_{j} \frac{c_{j i}-x_{1}}{b_{j}{ }^{2}}
$$

3) PID tuning of RBF network

Using the incremental PID controller, the control error is $e(k)=\theta_{r e f}-\theta_{r}$.

The input signals of PID controller as follows:

$$
\left.\begin{array}{c}
x_{1}(k)=e(k)-e(k-1) \\
x_{2}(k)=e(k) \\
x_{3}(k)=e(k)-2 e(k-1)+e(k-2)
\end{array}\right\}
$$

$e(k)$ is the error signal.

The principle diagram of the RBF neural network PID position controller is shown in Fig.3. 


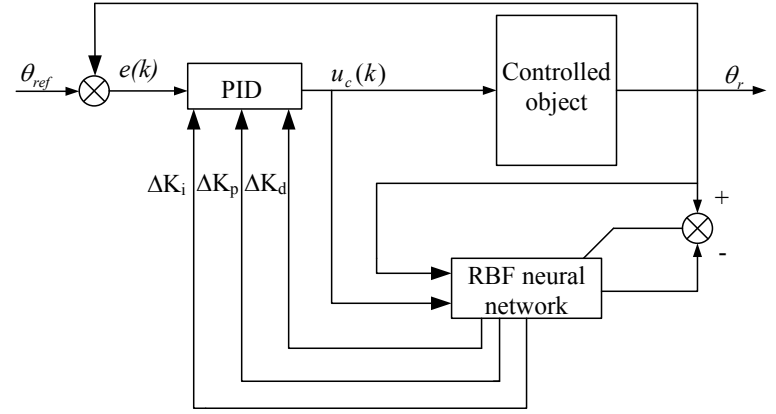

Figure 3. RBF neural network PID controller

RBF neural network PID controller can automatically adjust the corresponding weighting coefficient in realtime, can automatically adapt to the environment. Control algorithm is as follows:

$$
\left\{\begin{array}{r}
u_{c}(k)=u_{c}(k-1)+\Delta u_{c}(k) \\
\Delta u_{c}(k)=k_{p}\left(e(k)-e(k-1)+k_{i} e(k)+\right. \\
k_{d}(e(k)-2 e(k-1)+e(k-2)
\end{array}\right.
$$

$\mathrm{RBF}$ neural network regulating function as follows:

$$
J(k)=\frac{1}{2} e(k)^{2}
$$

Gradient descent method is used to $k_{p}, k_{i}, k_{d}$ adjustment:

$$
\left\{\begin{array}{l}
\Delta k_{p}=\eta_{k p} e(k) \frac{\partial y}{\partial \Delta u} x_{1}(k) \\
\Delta k_{i}=\eta_{k i} e(k) \frac{\partial y}{\partial \Delta u} x_{2}(k) \\
\Delta k_{d}=\eta_{k d} e(k) \frac{\partial y}{\partial \Delta u} x_{3}(k)
\end{array}\right.
$$

Where $\eta_{k p}, \eta_{k i}, \eta_{k d}$ are learning rate, $\frac{\partial y}{\partial \Delta u}$ is the Jacobi information for controlled object, can be obtained through the network identification.

The principle diagram of the controller is shown in Fig.4.

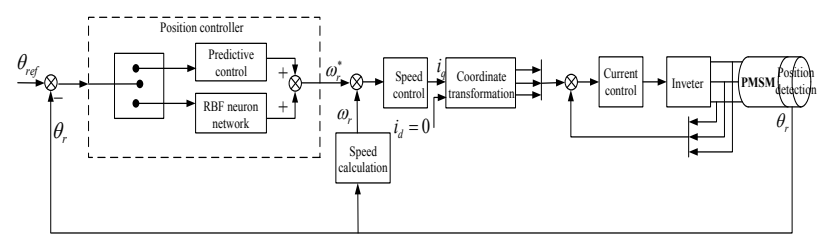

Figure 4. The principle diagram of the designed controller

\section{SIMULATION EXPERIMENTS}

According to the mathematical model, parameters are as follows: the motor and load moment of inertia $J=$ $0.00126 \mathrm{~kg} \cdot \mathrm{m}^{2}$; rated torque $6 \mathrm{~N} \cdot \mathrm{m}$, electromagnetic torque factor $K_{t}=1.0 \mathrm{~N} \cdot \mathrm{m} / \mathrm{A} ; \quad$ damping coefficient $B=0.000143 \mathrm{~N} \cdot \mathrm{m} \cdot \mathrm{s} ;$ stator resistance $R_{a}=1.21 \Omega$; winding inductance $L_{d}=L_{q}=0.00387 \mathrm{H}$; rated current $I_{e}=6.0 \mathrm{~A}$; motor pole logarithmic $P_{n}=4$; gear reduction ratio is $1: 152$.
Predictive controller parameters: control length $m=7$, predictive length $n=9$.

RBF neural network position controller parameter is: $\eta_{k p}=0.081, \eta_{k i}=0.0062, \eta_{k d}=0.0003$.

Traditional PID controller parameter $k_{p}=20, k_{I}=0.02$, $k_{D}=0.6$.

\section{A. Constant load disturbance}

Suppose to join a step disturbance $10 \mathrm{~N} \cdot \mathrm{m}$ at simulation time $1.2 \mathrm{~s}$, the response curves are shown in Figs. 5-6. The Fig.6 shows that the position response of traditional PID algorithm has a larger deviation and needs a longer recovery time. The Fig.5 shows the designed controller improves the system anti-interference ability.

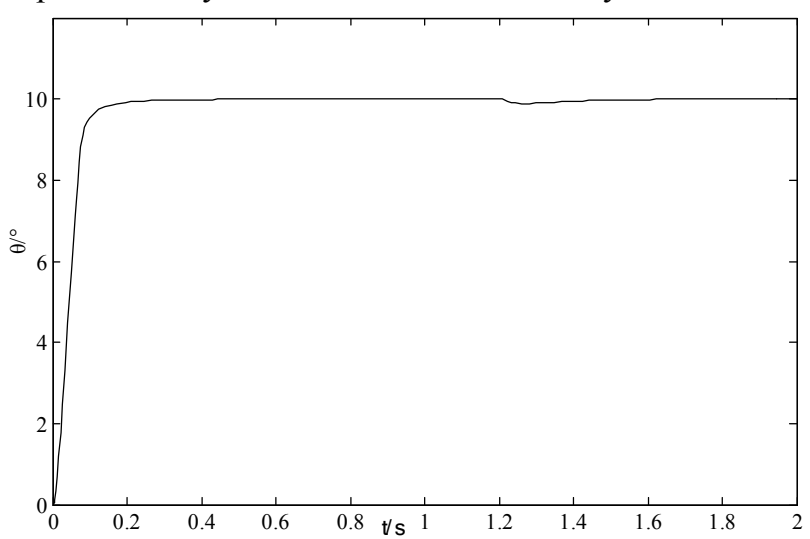

Figure 5. The response curve of the designed controller

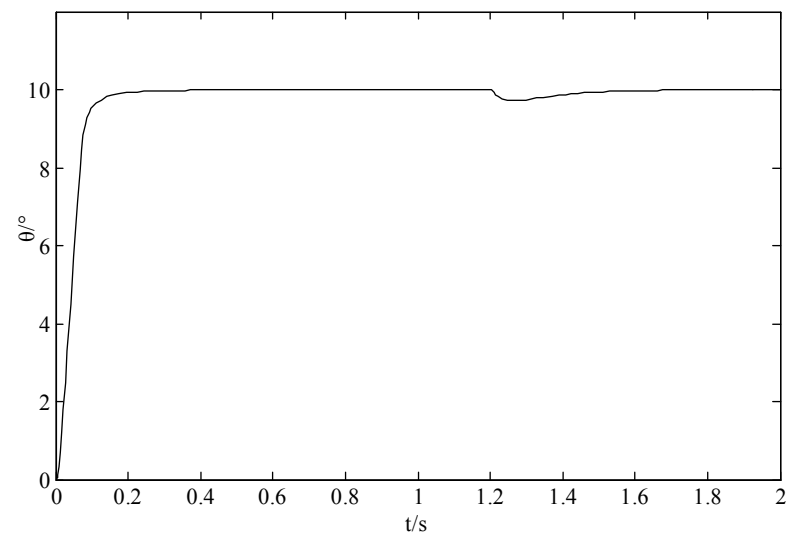

Figure 6. The response curve of the traditional controller

\section{B. The system parameter change}

In order to verify the control effect, it is assumed that the moment of inertia by $J=0.00126 \mathrm{~kg} \cdot \mathrm{m}^{2}$ into $J=0.00252 \mathrm{~kg} \cdot \mathrm{m}^{2}$. Fig. 7 shows the system response with no overshoot for designed control strategy. Fig. 8 shows that the system response is fast and small overshoot for the traditional PID control. 


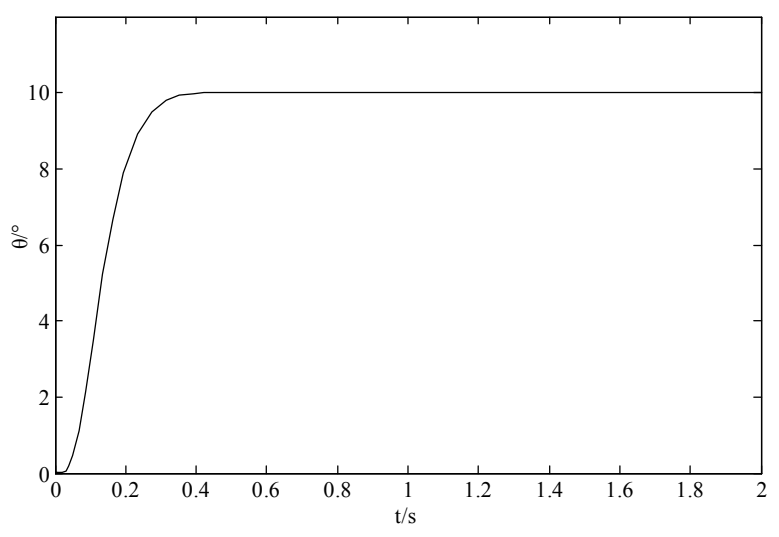

Figure 7. The response curve of the designed controller

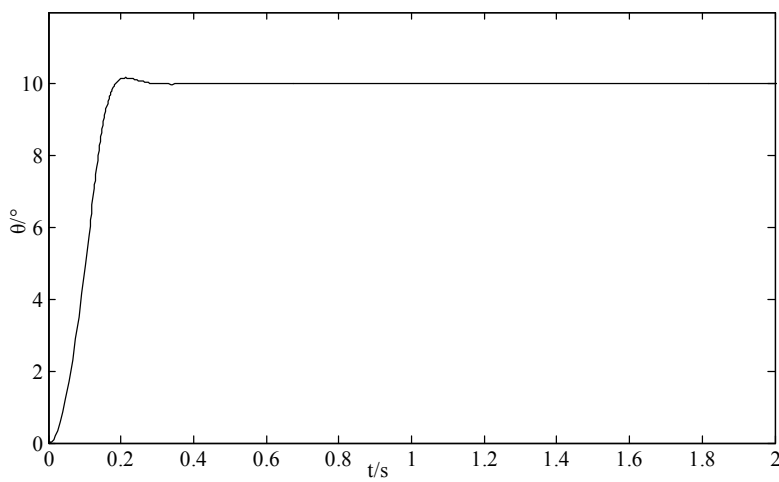

Figure 8. The response curve of the traditional controller

\section{The sinusoidal tracking experiment}

The target tracking function of the system is: $10 \sin (0.7166 \mathrm{t})$. The tracking error curves are shown in Figs. 9-10. As can be observed in the figures, the designed controller has better tracking properties compared to the traditional PID control.

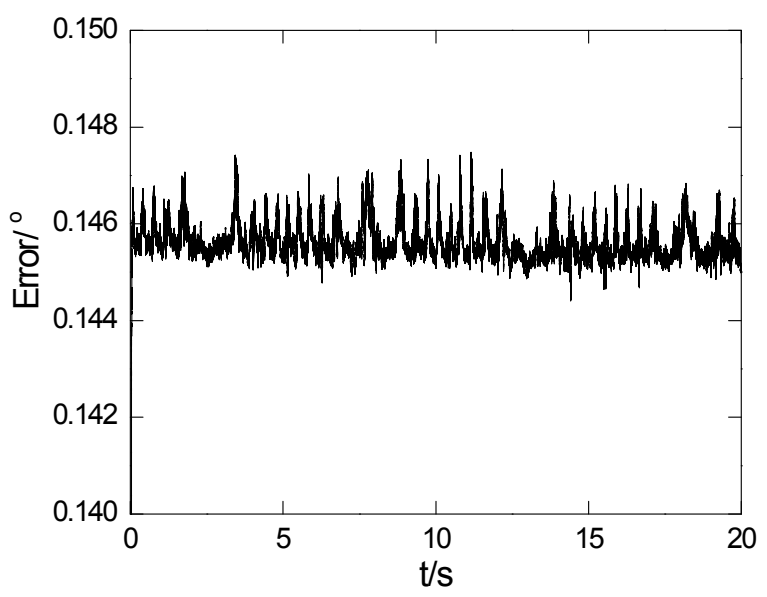

Figure 9. The error curve of traditional control

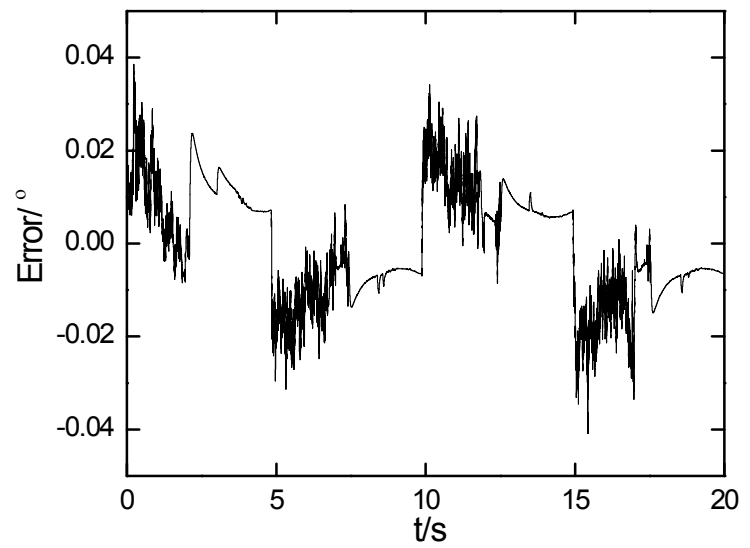

Figure 10. The error curve of designed controller

\section{CONCLUSIONS}

The paper study the position control of AC servo system, joint with predictive control and RBF neural network control, design the servo controller. The simulation experimental results demonstrate that when the system parameters change and external disturbance, designed control strategy can guarantee the system robustness.

\section{ACKNOWLEDGMENT}

The work is supported by the "A Project of Shandong Province Higher Educational Science and Technology Program (J13LB10)".

\section{REFERENCES}

[1] J.Q.Han, "From PID to Active Disturbance Rejection Control", IEEE Transactions on Industrial Electronics, Vol.56, (3), 2009 , pp.900-906.

[2] Li haixia, "Research on the Sensorless Fuzzy Vector Control Strategy for AC Servo System", Machine Tool \& Hydraulics, vol.41, (1), 2014, pp.31-34.

[3] Stumper,J.-F., Kuehl, S., Kennel, R, "Predictive torque control for AC drives: Improvement of parametric robustness using two-degreeof-freedom control", Energy Conversion Congress and Exposition (ECCE), 2013, pp.1170-1175.

[4] Liu meiqin, Liao xiaoxin, "Deng Yanni, Alternating Current Servo System by The Predictive Control Based on Model of The Time Delay RBFNN", Proceedings of the CSEE, vol.20, (1), 2000, pp.710.

[5] Wu lifen, Ma helin, Sun de, "Application of Neural Network Monitor Control Algorithm in the Servo System", System Simulation Technology, vol. 8, (2), 2012, pp.128-132.

[6] Dong weijie, Liu changhua, Song hua, "Application Contrast on Servo Electromotor Model between RBF and PIDNN", Control Engineering of China, vol.15,(5), 2008, pp.113-118.

[7] Xia changliang; Chen ziran; Xue mei, "Adaptive PWM Speed Control for Switched Reluctance Motors Based on RBF Neural", The Sixth World Congress on network intelligent Control and Automation ( WCICA ), vol.2, 2006, pp.8103-8107.

[8] Guo yajun, Wang xiaofeng, Ma dawei, Le guigao, "Application of Adaptive Fuzzy Sliding Mode Control to Rocket Coupling System", Journal of Nanjing University of Science and Technology, vol.36, (4), 2012, pp.618-622.

[9] Sun Yu, Wang Zhiwen, Kong Fanli, "Guidelines for the design of AC servo system", Machinery Industry Press, 2013.

[10] Mohammad Marufuzzaman, "Hardware Implementation of Current dq PI Controller for FOC PMSM Drive", LAP LAMBERT Academic Publishing, 2012. 